Ann. Biol. anim. Bioch. Biophys., I963, 3 (2), I9I-I99.

\title{
DOSAGE DE LA CYSTINE DANS LES ALIMENTS
}

\author{
Catherine de BELSUNCE et R. PION \\ Laboratoire d'Étude des Métabolismes, \\ Centre national de Recherches zootechniques, Jouy-en-Josas (Seine-et-Oise)
}

\section{SOMMAIRE}

La destruction de la cystine au cours de l'hydrolyse acide des protéines nécessite une détermination séparée de cet acide aminé.

Une oxydation à froid par l'acide performique transforme, au sein de la protéine, la cystéine et la cystine en acide cystéique.

Cet acide aminé, libéré par hydrolyse acide, est séparé par chromatographie soit sur échangeur de cations (Amberlite CG I 20) soit sur échangeur d'anions (Dowex 2), puis dosé par la ninhydrine.

Le choix des conditions expérimentales a été fait après avoir étudié les différentes étapes du dosage : l'oxydation, l'hydrolyse, le fractionnement et la colorimétrie.

Cette méthode d'oxydation est applicable à des aliments complets, sans extraction préalable des protéines.

Un certain nombre d'échantillons a été analysé; les résultats ont été comparés avec des données de la littérature, obtenues grâce aux méthodes chimiques, microbiologiques ou chromatographiques.

\section{I. - INTRODUC'TION}

Les acides aminés soufrés constituent le facteur limitant de beaucoup d'aliments protéiques. Or, 60 à $80 \mathrm{p}$. Ioo (suivant les animaux et les conditions physiologiques) du besoin en cystine et méthionine peuvent être couverts par la cystine. Le dosage de celle-ci dans les protéines alimentaires est donc particulièrement important.

Divers auteurs ont tout d'abord dosé la cystine à l'aide de méthodes chimiques (Lugg, I933 ; Hess, Sullivan et Palmes, I94I).

Depuis I950, elle a été dosée surtout par voie microbiologique, en utilisant en particulier Leuconostoc mesenteroides $P 60$ (CAmien et DUnN, I950; Chevilidard, FAUCONNEAU et Roche, I952) dans des conditions de milieu assez artificielles : absence d'acide aspartique et d'acide glutamique qui gênent la croissance. Cette méthode de dosage a été utilisée pendant une dizaine d'années sans possibilité de 
contrôle, car on ne connaissait pas d'autre microorganisme ou d'autre milieu pouvant servir au dosage de la cystine.

Les méthodes de dosage exigent que la cystine soit libre. Or, les procédés d'hydrolyse qui la libèrent risquent de la détruire, surtout en présence de glucides : c'est ce qui se produit au cours de 1'hydrolyse acide à l'aide d'HCl $6 \mathrm{~N}$ bouillant, utilisée pour doser les acides aminés par chromatographie sur colonne, d'après la méthode de MoORe, Spackman et STEIn (I958). Beaucoup d'auteurs utilisant la méthode microbiologique ont été conduits à recommander des hydrolyses de courte durée ( 6 à 8 heures : ChevillaRd et al.), ou effectuées à l'aide d'acide chlorhydrique peu concentré $(\mathrm{I}, 25 \mathrm{~N}$ : TISDALI, et al., I950).

Nous avons cherché à utiliser, pour le dosage de la cystine, la méthode chromatographique sur colonne qui présente de nombreux avantages (précision et spécificité en particulier).

En I954, Schram, MOORE et BIGWOOD, ont mis au point une transformation de la cystine en acide cystéique par oxydation performique au sein de la protéine. L'acide cystéique peut alors être libéré sans destruction par hydrolyse acide, et dosé par chromatographie sur colonne.

Mais ces auteurs ont observé une perte de cystine qu'ils ont estimée à Io p. Ioo, ce qui les a conduits à appliquer un facteur de correction.

La méthode a été modifiée par divers auteurs, en particulier HIRS, STEIN et MOORE (I954), HIRs (I956), BIDMEAD et LEY (I958), JunGE et al., (I959), JIRGENSONS, IKENAKA et GoRGURAKI (I960).

Nous avons cherché à obtenir un recouvrement quantitatif de cystine, et pou cela nous avons utilisé la méthode de Schram, MOORE et Bigwood (I954), modifié par BIDMEAD et LEY (I958), en étudiant les différentes étapes du dosage.

\title{
II. - MA'TÉRIEL E'T MÉ'THODES
}

\author{
I $\left.^{\circ}\right)$ Echantillons
}

Les analyses ont été faites sur des échantillons dans lesquels les protéines sont associées à des quantités variables de divers glucides : œufs, lait, farines de poisson, tourteaux, céréales, protéines de fourrage associées aux constituants membranaires : résidus d'extraction alcoolique à $0^{\circ} \mathrm{C}$ (traitement A) ou d'extraction alcoolique suivie d'extraction aqueuse à $0^{\circ} \mathrm{C}$ (traitement $\mathrm{B}$ ).

\section{$\left.2^{0}\right)$ Oxydation de la protéine}

Réactifs :

- Acide formique pur $99 \mathrm{p}$. Ioo conservé en flacon brun (Prolabo).

- Perhydrol 33 p. Ioo conservé à $+4^{\circ} \mathrm{C}$ (Merck).

- Méthanol pur (Prolabo).

L'acide performique est préparé à partir de 95 volumes d'acide formique $99 \mathrm{p}$. 100 à $0^{\circ} \mathrm{C}$, auxquels on ajoute 5 volumes d'eau oxygénée $33 \mathrm{p}$. 100 à $0^{\circ} \mathrm{C}$ et 0,5 volume de méthanol. Après deux heures à la température ambiante et à l'obscurité, le mélange est refroidi à $-10^{\circ} \mathrm{C}$ et versé dans un ballon contenant l'échantillon à analyser. Le ballon est maintenu à $-10^{\circ} \mathrm{C}$ pendant 4 heures ou plus, à l'abri de la lumière. La réaction est arrêtée par addition de 2 volumes d'eau glacée. L'acide performique est éliminé par 5 ou 6 évaporations et reprises successives à l'eau, dans un évaporateur rotatif sous vide, en évitant d'aller à sec (source chaude $38-40^{\circ} \mathrm{C}$, source froide $-18,-20^{\circ} \mathrm{C}$ ). 


\section{$\left.3^{0}\right)$ Hydrolyse acide}

Réactif : HCI (RP Prolabo).

On ajoute au résidu de l'évaporation, dans le même ballon, $500 \mathrm{ml}$ d'HCl $6 \mathrm{~N}$; on adapte un réfrigérant à reflux, et le ballon est immergé dans un bain d'huile à $125^{\circ} \mathrm{C}$ pendant 24 heures.

L'acide chlorhydrique est éliminé le plus complètement possible, à l'aide de la même technique et dans les mêmes conditions que l'acide performique. Le résidu final est repris, amené au $\mathrm{pH}$ et au volume désirés.

Réactifs :

\section{$\left.4^{\circ}\right)$ Séparation chromatographique}

- Amberlite CG 20.

- Dowex $2 \times 10,200-400$ mesh (Bio-Rad).

- Tampons citrate de sodium (cf. MoORe et Stein, I958).

- Acide monochloracétique RP cristallisé (Prolabo) conservé en flacons bruns à $+4^{\circ} \mathrm{C}$.

- Brij 35.

a) Sur échangeur de cations: une fraction aliquote de l'hydrolysat ( $5 \mathrm{ml}$ au maximum), préalablement amené ’̀ $\mathrm{pH} 2$ dans un tampon citrate de sodium $0,2 \mathrm{~N}$, est posée sur une colonne de I $50 \times 0,9 \mathrm{~cm}$ d'Amberlite $\mathrm{CG}_{12}$. L'élution est effectuée avec du tampon citrate de sodium o, $2 \mathrm{~N}$ de $\mathrm{pH}$ 3,25 (cf. MOORE et Stein, I958) à $50^{\circ} \mathrm{C}$. On recueille 80 fractions de $\mathrm{I} \mathrm{ml}$, le pic d'acide cystéique se trouvant entre la $3^{\circ}$ et la $40^{\circ}$ fraction. La colonne est ensuite régénérée par passage de $\mathrm{NaOH}$ $0,2 \mathrm{~N}$, puis équilibrée par du tampon citrate de $\mathrm{pH} 3,25$.

b) Sur échangeur d'anions: une fraction aliquote de l'hydrolysat, amené à $\mathrm{pH} 5$ avec de la soude, est posée sur une colonne de $15 \times 0,9 \mathrm{~cm}$ de Dowex $2 \times 8,200-400$ mesh (cf. Schram, Moore et BIGWOOD, 1954). L'élution est effectuée à la température ambiante $\left(20^{\circ} \mathrm{C}\right)$ par des solutions d'acide monochloracétique contenant $5 \mathrm{ml}$ par litre de Brif 35. Après 60 à $70 \mathrm{ml}$ d'acide monochloracétique $0, \mathrm{I} \mathrm{N}$, on utilise de l'acide monochloracétique $0,3 \mathrm{~N}$ pour éluer l'acide cystéique en un nombre minimum de fractions que l'on recueille à l'aide d'un collecteur automatique (80 fractions de I $\mathrm{ml}$ ).

La régénération se fait par $50 \mathrm{ml}$ d'acide monochloracétique $\mathrm{N}$ suivis de $100 \mathrm{ml}$ d'acide $\circ, \mathrm{I}$.

\section{$\left.5^{\circ}\right)$ Dosage colorimétrique}

Les fractions recueillies sont dosées avec le réactif à la ninhydrine de Moore et STEiN (I954), en utilisant I $\mathrm{ml}$ de réactif par fraction de I à $\mathrm{I}, 2 \mathrm{ml}$. La densité optique est lue au colorimètre Lumetron (filtre $55^{\circ} \mathrm{m} \mu$ ).

\section{III. - RÉSUL'TA'TS}

Les différentes étapes du dosage ont été étudiées afin de déterminer les meilleures conditions expérimentales.

\section{Io) Séparation chromatographique}

Les deux procédés de chromatographie ont été comparés.

a) Les taux de recouvrement ont été déterminés avec des solutions standard d'acide cystéique. Les valeurs figurant sur le tableau $I$ font apparaître un bon recouvrement.

b) Des hydrolysats de différents aliments ont été chromatographiés simultanément sur Amberlite CG I20 et sur Dowex 2. Les résultats (tab1. 2) sont très comparables. 
Le fractionnement sur Dowex 2 a été retenu, car il est plus facile à utiliser (petites colonnes, à la température ambiante), et ne nécessite pas d'ajustement précis du $\mathrm{pH}$ des solutions éluantes. De plus, le volume de la prise d'essai n'a pas d'influence sur le pic d'acide cystéique. Au contraire, si l'on pose ro ml d'échantillon sur la colonne d'Amberlite, on observe un pic dissymétrique qui peut faire croire à la présence d'un composé interférent.

\section{TABLEAU I}

Influence du mode de fractionnement sur le recouvrement d'acide cystéique

\begin{tabular}{|c|c|c|}
\hline Mode de fractionnement & $\begin{array}{c}\% \text { ac. cys- } \\
\text { téique retrouv'é }\end{array}$ & $\begin{array}{c}\text { Nombre de } \\
\text { fractionnements }\end{array}$ \\
\hline $\begin{array}{l}\text { Sur Amberlite } \\
\text { CG } 120 \text { torme } \mathrm{Na}^{+} \ldots \ldots\end{array}$ & 94,5 & 2 \\
\hline 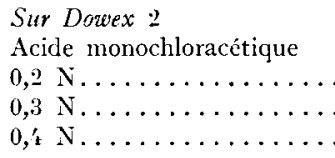 & $\begin{array}{r}97 \\
101 \\
97\end{array}$ & $\begin{array}{l}2 \\
3 \\
2\end{array}$ \\
\hline
\end{tabular}

TABLEAU 2

Influence du mode de fractionnement sur les résultats obtenus pour quelques échantillons: cystine en $g / 16$ g $N$

\begin{tabular}{|c|c|c|}
\hline Échantillcus & $\begin{array}{l}\text { Sur Amberlite } \\
\text { CG } 120\end{array}$ & Sur Dowex 2 \\
\hline Maïs $\ldots . . . .$. & $\begin{array}{l}2,1 \mathbf{1} \\
2,5\end{array}$ & $\begin{array}{l}2,4 \\
2,45\end{array}$ \\
\hline Euf entier. . . . . . . & 2,8 & 2,7 \\
\hline Ovalbumine & 1,85 & 1,75 \\
\hline $\begin{array}{l}\text { Protéine de luzerne } \ldots \ldots \ldots \\
\quad(\text { Traitement } B) \ldots \ldots \ldots\end{array}$ & $\begin{array}{l}1,45 \\
1, ;\end{array}$ & $\begin{array}{l}1,3 \\
1,35\end{array}$ \\
\hline
\end{tabular}

20) Oxydation

a) Test de recouvrement. - Le rendement de la transformation de la cystine pure oxydée en acide cystéique est de $95 \mathrm{p}$. Ioo en moyenne (oxydation de 4 heures à - IO ${ }^{\circ} \mathrm{C}$, suivie d'une hydrolyse de 24 heures).

b) Temps de réaction. - Plusieurs échantillons d'aliments assez différents ont été oxydés pendant 4 et $\mathrm{I} 6$ heures. Les résultats exprimés en $\mathrm{g}$ de cystine pour Ioo $\mathrm{g}$ 
de protéines $(\mathrm{N} \times 6,25)$ se trouvent sur le tableau 3. L'identité des valeurs obtenues a permis d'adopter le temps de 4 heures, en vérifiant que la température ne descend pas au-dessous de - IO ${ }^{\circ} \mathrm{C}$ (à cause du risque de congélation) et ne dépasse pas $-5^{\circ} \mathrm{C}$ (dans ce cas il peut se produire une perte de cystine).

TABIEAU 3

Infuence du temps d'oxydation: cystine en $g / 16 \mathrm{~g} N$

\begin{tabular}{|c|c|c|c|c|c|}
\hline $\begin{array}{c}\text { Temps } \\
\text { d'oxydation }\end{array}$ & Euf & Mais & Ovalbumine & Lait en poudre & $\begin{array}{l}\text { Farine de } \\
\text { poisson }\end{array}$ \\
\hline 4 heures.... . & 2,55 & 2,5 & 1,85 & 1,0 & 1,15 \\
\hline 16 heures & 2,5 & 2,4 & 1,8 & 0,9 & 1,1 \\
\hline
\end{tabular}

\section{$\left.3^{\circ}\right)$ Réaction à la ninhydrine}

Le facteur de coloration de l'acide cystéique par rapport à la leucine $(0,92)$ est le même qu'il soit obtenu en utilisant le tampon '3,25, 1'acide monochloracétique $0,2 \mathrm{~N}$, ou l'acide monochloracétique $0,3 \mathrm{~N}$. En revanche, il est plus faible et surtout plus irrégulier si on utilise l'acide monochloracétique $0,4 \mathrm{~N}$; en effet, la plus forte concentration en acide risque de dépasser le pouvoir tampon du réactif ; c'est pourquoi la concentration $0,4 \mathrm{~N}$ n'a pas été utilisée.

Une solution standard d'acide cystéique est utilisée comme témoin de coloration.

\section{$\left.4^{\circ}\right)$ Application à différents aliments}

Nous donnons sur le tableau 4 les résultats obtenus avec un certain nombre de produits couramment employés en alimentation animale.

\section{VI. - DISCUSSION}

Sur le tableau 4 nous avons reporté, outre nos propres résultats, un certain nombre de valeurs obtenues par les trois méthodes de dosage. Nos résultats s'accordent assez bien avec ces données pour des produits tels que le lait, les protozoaires et bactéries du rumen, certains tourteaux. Ils s'expriment par des valeurs légèrement plus faibles pour les farines de poisson, un peu plus élevées pour l'œuf.

Les différences sont plus importantes pour l'ovalbumine d'une part, et les produits végétaux d'autre part.

Dans le cas de l'ovalbumine nous avons un résultat de cystine beaucoup plus faible; ceci pourrait être dû au fait que le produit commercial utilisé a pu subir des modifications au cours de sa fabrication. 
C. de BELSUNCE, R. PION

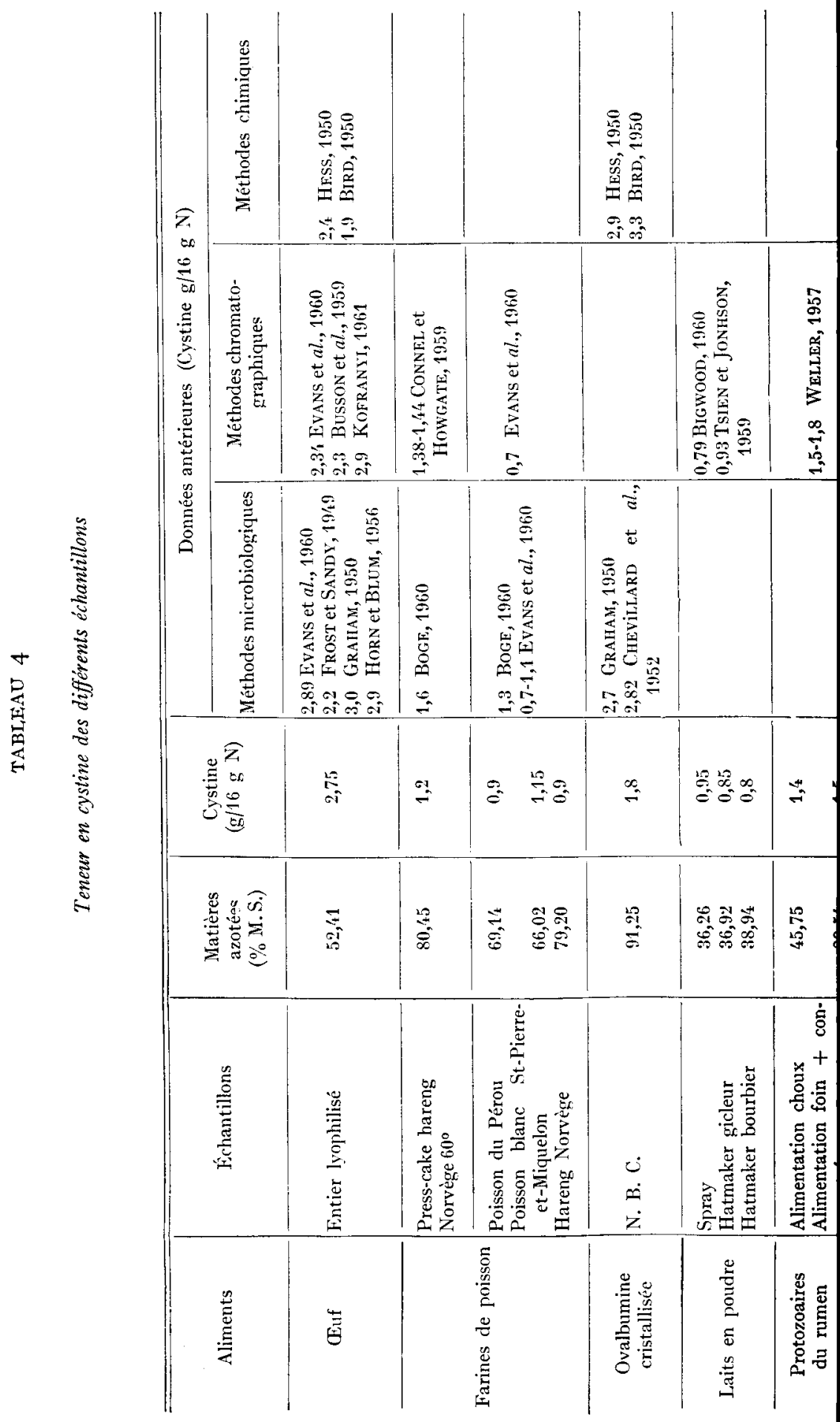




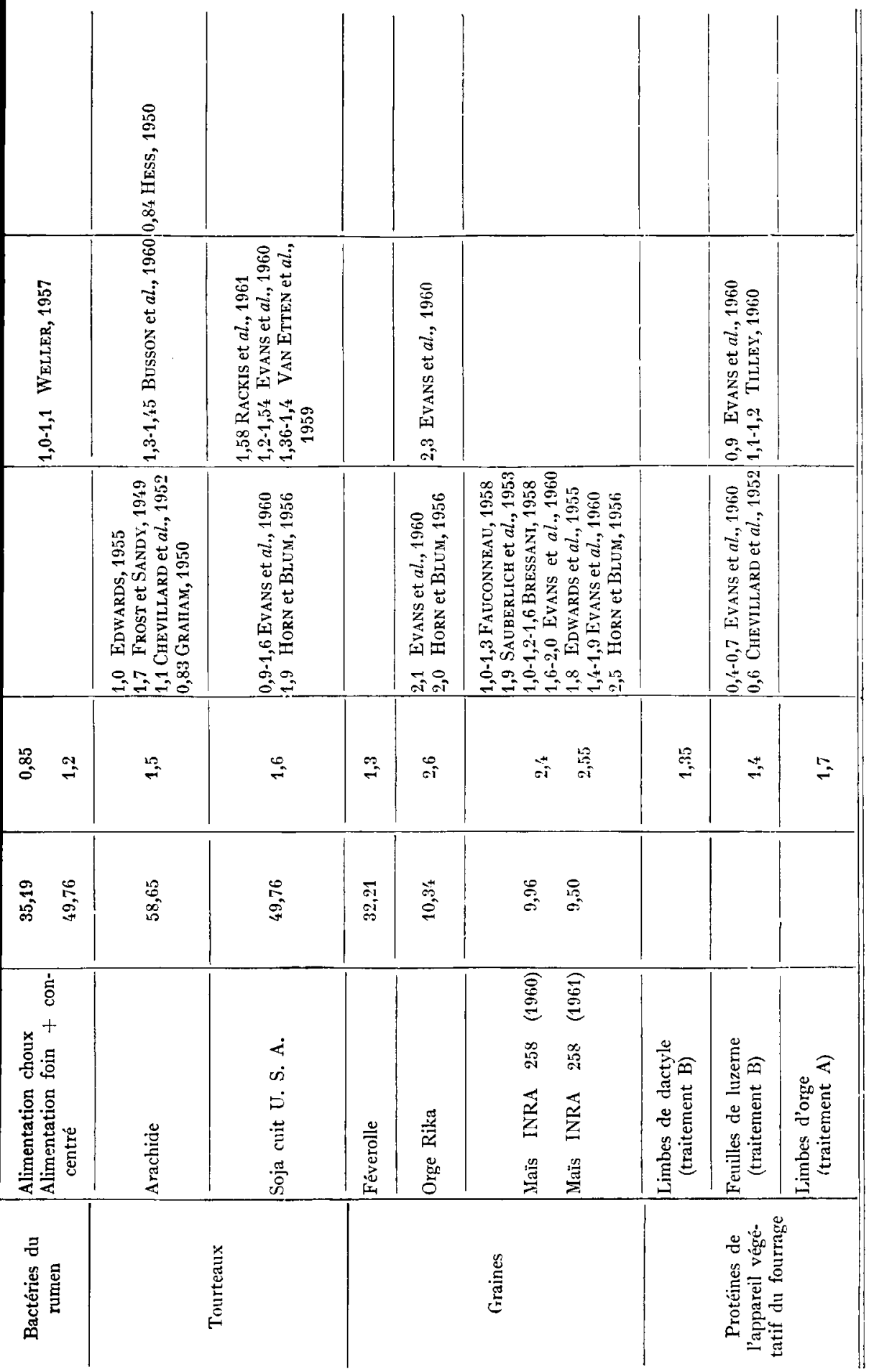


En ce qui concerne les fourrages et surtout les graines, nos résultats sont nettement plus élevés que ceux que donnent les méthodes microbiologiques. Ceci pourrait s'expliquer par la présence dans les produits végétaux de quantités importantes d'acide aspartique et d'acide glutamique (de l'ordre de $30 \mathrm{p}$. Ioo) qui gênent la croissance du Leuconostoc mesenteroides, alors que le milieu de Camren et Dunn n'en contenait pas. D'autre part, EVANS, BANDEMER et BAUER (I960) ont obtenu des résultats très variables en fonction du temps d'hydrolyse : les temps très courts (30 mn) semblent donner des valeurs trop élevées, ce qu'ils expliquent par une action des peptides sur la croissance du microorganisme. Les résultats trop faibles obtenus à la suite d'hydrolyses plus longues sont probablement dus à la destruction de la cystine au cours de l'hydrolyse.

La méthode d'oxydation préalable de la protéine paraît plus sûre car elle permet de transformer la cystine en un composé stable et ainsi d'obtenir une récupération plus quantitative.

\section{Rę̧u pour publication en mars 1963.}

\section{SUMMARY}

\section{DETERMINATION OF CYSTINE IN FOOD-STUFFS}

Cystine is partially destroyed in the course of acid hydrolysis used for the determination of other amino acids. To avoid this destruction the method of SCHRAM, MoORE and Bigwood (1954) has been used, which consists of oxidysing the cystine within the protein, into cysteic acid, which is resistant to acid hydrolysis. In order to obtain a quantitative yield, the reagent of BIDMEAD and LEY (1958), at $-10^{\circ} \mathrm{C}$ was used. Cysteic acid is liberated by acid hydrolysis, separated by column chromatography, either on anion exchange resin (Dowex II) or on cation echange resin (Ámberlite CG I20), and determined colorimetricaly with ninhydrine (MOORE and STEIN, I954).

The different steps of the determination were studied :

- Chromatography : the two methods were compared, either with standard solutions of cysteic acid (table I), or with hydrolysates obtained from different samples (table 2). The results obtained by the two methods are very similar. The use of the Dowex II was preferred for practical reasons.

- Oxydation : the average yield of the transformation of pure cystine into cysteic acid is 95 p. I0o. A study of the time of the reaction (table 3) made it possible to adopt a reaction time of 4 hours.

Reaction with ninhydrine : the factor of coloration of cysteic acid is the same in the different eluants tried, except for monochloracetic acid $0.4 \mathrm{~N}$. So the use of the latter was discarded.

The results obtained for the different feeds (table 4) agree generally with those obtained by other authors using similar methods, but sometimes differ considerably (particularly for cereals) from the results of microbiological determinations. These may be either too low, if the growth of the microorganism is inhibited by the presence of an excess of aspartic and glutamic acid, or too high, if certain products of incomplete hydrolysis behave like growth factors.

\section{RÉFÉRENCES BIBLIOGRAPHIQUES}

Bidmead D. S., Ley F. J., 1958. Quantitative amino acid analysis of food proteins by means of a single ion-exchange column. Biochim. Biophys. Acta, 29, 562-567.

Bigwood E. J., I960. Travaux du Comité pour l'étude des maladies et de l'alimentation du bétail. I.R.S. I. A. I. W. O. N. L. Comples rendus de recherches, 26, 62.

BiRd O. D., I950. Cité par Rutgers University : Cooperative determinations of the amino acid content and of the nutritive value of six selected protein sources. 
Bressani R., SCRIMSHAw N. S., 1958. Effect of lime treatment on in vitro availability of essential amino acids and solubility of protein fractions in corn. J. Agric. Food Chem., 6, 774-778.

Busson F., Carbiener R., I3ergeret B., 1959. Étude de la fraction protidique des graines de Vigna Unguiculata. Qual. Plant. Materiae vegetabiles, 6, I I-15.

Boge G., 1960. Amino acid composition of herring (clupea harengus) and herring meal. Destruction of amino acids during proceessiny. J. Sci. Fd Agric., 11, 362-365

Camien M. N., DunN M. S., i950. An improved microbiological method for the determination of cystine in human urine with Leuconostoc mesenteroides P. 6o. J. Biol. Chem., 183, 561-568.

Chevillard L., Fauconneau G., Roche J., i952. Dosage microbiologique de la cystine. Application aux produits végrétaux utilisés dans l'alimentation du bétail. Ann. Zootech., 3, r-I3.

Connel J. J., Howgate P. F., I959. The amino acid composition of some british food fishes. J. Sci. Fd Agric., 10, 24I-244.

Dunn M. S., 1949. Determination of amino acids by nicrobiological assay. Physiol. Rev., 29, $29 \mathrm{r}$.

Dustin J. P., Schram E., Moore S., Bigwood E. J., 1953. Dosage chromatographique des acides aminés d'une graine de céréale (orge), d'un foin et d'un tourteau de lin. Bull. Soc. Chim. Biol., 35, I I 37-1 I47.

Edwards C. H., Carter C. P., 1955. Cystine, tyrosine and essential amino acids of selected foods. J. Agric. Food Chem., 3, 952-957.

Evans R. J., Bandemer S. L., Bauer D. H., ig6o. Amino acid measurement, cystine content of proteins, foods and feeds. Comparison of chromatography on a sulfonated polystyrene resin and microbiologrical methods of determination. J. Agric. Food. Chem., 8, 383-386.

Fauconneau G., I957-1958. Non publié.

Frost D. V., SAndy H. R., 1949. Assay of dry proteins by rat repletion method. Nutritive value and amino acid composition of six reference proteins. J. Nutr., 39, 427-439.

Graitam C. E., r950. Cité par Rutgers University : Cooperative determinations of the amino acid content and of the nutritive value of six selected protein sources.

Hess L. M., Sullivan M. K., Palmes E. D., I94I. The colorimetric determination of cystine in tobacco mosaic virus protein. Proc. Soc. Exp. Biol. Med., 48, 353.

Hess W. C.. 1950. Cité par Rutgers University : Cooperative determination of the amino acid content a of the nutritive value of six selected protein sources.

Hirs C. H. W., Stein W. II., Moore S., I954. The amino acid composition o ribonuclease. J. Biol. Chem., 211, 941-950.

Hirs C. H. W., i956. The oxydation of ribonuclease with performic acid. J. Biol. Chem., 219, 6r I-62 I.

Horn M. J., Blum A. E., 1956. Cité par Evans R. J. et al. J. Agric. Food Chem., 8, 383-386.

Jirgenjons B., Ikenaka T., Gorguraki V., ig6o. Chemical studies on a macroglobulin. Clin. Chim. Acta, 5, 502-507.

Junge J. M., Stein E. A., Neurath H., Fisher F. H., i959. The amino acid composition of amylase from Bacillus subtilis. J. Biol. Chem., 234, 556-56.

Kofrany E., Müller Wecker H., i96. Der Einfluss des nichtessentiellen Stickstoffs auf die biologische Wertigkeit von Proteinen und die Wertigkeit von Kartoffelproteinen. Hoppe-Seyl. Z., 325, 60-64.

LUGG J. W., 1933. Sullivan's reaction for the quantitative determination of cysteine and cystine. Biochem. J., 27, 669 et I022.

MoOre S., Stein W. H., 1954. A modified ninhydrin reagent for the photometric determination of amino acids and related compounds. J. Biol. Chem., 211, 907-9I 3 .

Moore S., Spackman D. H., Stein W. H., 1958. Chromatography of amino acids on sulfonated polystyrene resins. An improved system. Anal. Chem., 30, I I $85^{-1}$ I 90.

Rackis J. J., Anderson R. L., Sasaine H. A., Smith A. K., Van Etten C. H., ig6i. Amino acids in soybean Hulls and oil meal fractions. J. Agric. Food Chem., 9, to9-412.

Sauberlicil M. E., Wan Yuin Chang, Salmon W. D., 1953. The amino acid and protein content of corn as related to variety and nitrogen fertilization. J. Nutr., 51, $241-250$.

Schram E., Moore S., BIgwood E. J., I954. Chromatographic determination of cystine as cysteic acid Biochem. J., 57, 33-37.

"Tilley J. M., J959-1960. Grassland Research Insl. Experiments in progress, 13, 8r.

Tisdall S. L., Davis R. I., Kingsley A. F., Mertz E. T., io5o. Methionine and cystine content of two strains of alfalfa as influenced by different concentrations of the sulfate ion. Agronomy J. New York, 2, $22[-225$.

Tsien W. S., Jonhson B. C., I959. Effect of radiation sterilization on the nutritive value of foods. V. On the amino acid composition of milk and beef. J. Nulr., $69,45^{-48}$.

Van Etten C. H., Hubbard J. F., Mallan J. M., Smith A. K., Blessin C. W., i959. Amino acid composition of soybean protein fractions. J. Agric. Food Chem., 7, 129-1 31 .

WELler R. A., 1957. The amino acid composition of hydrolysates of microbial preparations from the rumen of sheeps. Austr. J. Biol. Sci., 10, 384-389. 